Research Article

\title{
Direct Current Magnetron Glow Discharge Plasma Characteristics Study for Controlled Deposition of Titanium Nitride Thin Film
}

\author{
Sankar Moni Borah \\ Department of Physical Science, Gauhati University, Jalukbari, Guwahati, Assam 781014, India \\ Correspondence should be addressed to Sankar Moni Borah; sankarmoni@gmail.com
}

Received 31 January 2013; Accepted 18 April 2013

Academic Editor: Rodrigo Martins

Copyright (C) 2013 Sankar Moni Borah. This is an open access article distributed under the Creative Commons Attribution License, which permits unrestricted use, distribution, and reproduction in any medium, provided the original work is properly cited.

\begin{abstract}
This paper reports on the study of direct current (DC) magnetron glow discharge plasma characteristics in a cylindrical magnetron system in argon-nitrogen. Presence of nitrogen gas makes the plasma environment reactive, and it results in significant changes of the plasma properties - number density, electron temperature, floating potential, and sheath thickness. Applied magnetic field is a parameter which is closely related to proper deposition of thin film. Cylindrical Langmuir probe and Emissive probe are used as diagnostics for the estimation of various plasma parameters indicated earlier. Controlled titanium nitride (TiN) thin film deposition on bell-metal at different argon-nitrogen gases ratio is another important study reported.
\end{abstract}

\section{Introduction}

In today's modern civilization, plasma finds application as light sources, new kinds of television screens, in reactors for fusion experiments. The most common and probably of most economic importance is its application in material processing of solids and gases. Plasmas are industrially useful because they possess at least one of the following two important characteristics. The first characteristic is high power or high energy density. The second major important characteristic of the processing plasmas is that they produce active species which are more numerous, different in kind, and/or more energetic than those produced in chemical reactors. These active species make it possible to do things to the surface of materials that can be done in no other way. Such industrially useful active species are more frequently produced by corona or glow discharge plasmas, which have power densities in the range from below $10^{-4}$ to tens of watts per cubic centimeter. A large number of technological fields employ direct current magnetron glow discharge process for sputter deposition of different compound films [1,2]. This process is widely regarded as an environment friendly process since it fully avoids the use of various chemical reactants [3]. Magnetron glow discharge systems differ from the conventional glow discharge systems by employing externally applied magnetic field in axial direction parallel to the cathode surface. The electric field $\mathbf{E}$ has radial direction, and, hence, the charged particles in the cylindrical magnetron discharge move under the influence of the $\mathbf{E} \times \mathbf{B}$ field [4]. The externally applied magnetic field traps the energetic electrons and effectively increases the ionization efficiency resulting in high deposition rate at a relatively low pressure $[5,6]$, thereby making it a favourable process for many manufacturing applications. Generally, films are grown under low pressure to reduce collision events for higher surface mobility to ensure better quality of the deposited films [7-9]. In many plasma processing applications, magnetron reactive sputtering is actively used where metal oxides or metal nitride films are required for surface treatment. However, due to complex discharge behavior of the molecular gases and complexity generated by the various plasma species under the influence of crossed magnetic and electric fields, the basic physical mechanism in reactive magnetron sputtering is not fully understood. Furthermore, the delicate dependence of plasma parameters (density profile, temperature, and energy distributions) on the sputtering efficiency as well as on the physicochemical processes leading to quality film growth in reactive sputtering is undoubtedly a subject of investigation. 
Langmuir probe which facilitates the local estimation of the plasma parameters like plasma density, electron temperature, and floating potential at the probe position has been used. For the study of sheath and presheath behaviour, Emissive probe has been used. Titanium nitride (TiN) is a rock-salt structure $(\mathrm{NaCl})$ compound consisting of titanium atoms filled in face centered cubic (fcc) based lattice with all octahedral sites filled with nitrogen atoms. Due to its unique properties like high hardness, good wear, and corrosion resistance, TiN has been used as a coating for cutting tools or as an anticorrosive coating for turbines as in aerospatial industries [10]. Its lustrous golden yellow coloured film can be used for decorative applications.

\section{Experimental Setup}

The experimental magnetron device is a stainless steel cylindrical chamber having dimensions of $100.0 \mathrm{~cm}$ in length and $30.0 \mathrm{~cm}$ in diameter. A small hollow cylinder of titanium is placed coaxially inside the chamber which acts as the cathode. The length of the cathode is $25.0 \mathrm{~cm}$ and its outer diameter is $3.25 \mathrm{~cm}$. Both ends of the cathode have end reflectors of $5 \mathrm{~cm}$ in diameter. A schematic diagram of the experimental setup along with the probes and accessories is shown in Figures 1(a) and 1(b). For generation of steady axial magnetic field, two coils of enamel-coated copper (SWG 9) are placed around the body of the chamber. Each coil having 1500 turns is mounted over rails, which covers a length of $20.0 \mathrm{~cm}$ on the chamber axis and fitted with castor wheels so that it can be easily moved along the axis of the chamber for adjustment of the distance between the coils. Direct current is passed through both coils in the same direction which produces an axial magnetic field parallel to the cathode surface that is uniform within a length of $\sim 40.0 \mathrm{~cm}$ at the central portion of the chamber. The radial variation of the magnetic field is within $5 \%$.

The vacuum unit consists of a rotary pump and a diffusion pump. The base pressure of the chamber is brought down to $\sim 2 \times 10^{-6}$ Torr. The total partial pressure of the working gas mixture is maintained in the range of $\sim 1$ to $3 \times 10^{-3}$ Torr. Argon and nitrogen are injected into the chamber through a double valve system. A Pirani gauge and an ionization gauge are used for measurement of pressure inside the chamber. The discharge power is supplied from a stabilized DC power supply ( $1500 \mathrm{~V}, 5 \mathrm{~A})$ working in the voltage-regulated mode. Plasma is produced by applying DC voltage between cylindrical titanium electrode as cathode and the grounded chamber as anode. Typical discharge current is $100.0 \mathrm{~mA}$ to $250.0 \mathrm{~mA}$. Plasma parameters are measured with a cylindrical Langmuir probe made of tungsten having diameter $0.05 \mathrm{~cm}$ and length $0.5 \mathrm{~cm}$. Typical measured plasma parameters are plasma density $\sim\left(10^{9}-10^{10}\right) \mathrm{cm}^{-3}$ and electron temperature $\sim$ $(1.0-4.0) \mathrm{eV}$.

The radial plasma potential profiles are recorded with the help of an Emissive probe $\left(E_{p}\right)$, made of $1 \%$ thoriated tungsten wire of $0.005 \mathrm{~cm}$ in diameter and $0.3 \mathrm{~cm}$ in length. The probe wire is looped into a semicircle and is suitably attached at the two ends with two supporting stainless steel electrodes (each $0.025 \mathrm{~cm}$ in diameter). The supporting electrodes are covered by ceramic tubes for proper insulation with the plasma. The filament wire is heated by passing DC current through it until maximum electron emission occurs. Due to the emission of electrons, the floating potential of the probe increases towards positive value until it becomes equal to the plasma potential where it saturates. The resistance used for floating potential measurement is $30.0 \mathrm{M} \Omega$, and accordingly the potential value is calibrated in the $X-Y$ recorder (Yokogawa make). The continuous measurement of plasma potential profile is done by moving the probe slowly $(1.0 \mathrm{~cm} / \mathrm{min})$ using a motor driving system attached to the Emissive probe shaft.

\section{Experimental Results and Discussion}

The discharge current-voltage characteristics for different applied magnetic field strengths are shown in Figure 2. The total gas pressure has been maintained at $2 \times 10^{-3}$ Torr. With increasing discharge voltage, the discharge current increases satisfying the current-voltage relation,

$$
I \propto V_{d}^{n}
$$

where $I$ is the discharge current, $V_{d}$ is the discharge voltage, and $n$ is the index of the performance of the system. When the magnetic field is increased, the discharge current is found to increase for a fixed discharge voltage showing that at higher magnetic fields due to higher confinement high density plasma is achieved at relatively lower discharge voltage. At a discharge voltage of $600 \mathrm{~V}$, the discharge current is $64 \mathrm{~mA}$ at a magnetic field of 0.0075 Tesla, and it rises to $345 \mathrm{~mA}$ at a magnetic field of 0.015 Tesla. The value of $n$ ranges from 4.5 to 7 at magnetic field strengths between 0.01 Tesla and 0.0225 Tesla. This suggests that the system operates in the magnetron mode in the previous applied magnetic field strength range. For lower and higher magnetic field strengths (0.0075 Tesla and 0.025 Tesla, resp.), $n$ has a value less than three $(<3)$.

The measured plasma densities for different magnetic fields are shown in Figure 3 with probe position as a parameter. The total gas pressure is $2 \times 10^{-3}$ Torr and the discharge voltage is $600 \mathrm{~V}$. With increasing magnetic field, the electrons are confined closer to the cathode. The cyclotron frequency increases significantly, and the diffusion coefficient reduces across the magnetic field which decreases the particle loss rate. On the other hand, due to the $\mathbf{E} \times \mathbf{B}$ drift, the lifetime and also the path length of the electrons around the cathode increase. This type of electron motion increases the collision probability between the electrons and the atoms; therefore, the ionization rate increases. The resultant effect is that the plasma density becomes very high in the vicinity of the cathode due to enhanced ionization rate and lower diffusion rate. As the plasma density increases, the current density at the cathode becomes higher, and, therefore, the sputtering rate also increases. Again, as most of the electrons remain entrapped within the vicinity of the cathode and only the low velocity electrons are present beyond this magnetic entrapment, the plasma density reduces at larger radial distance away from the cathode. 


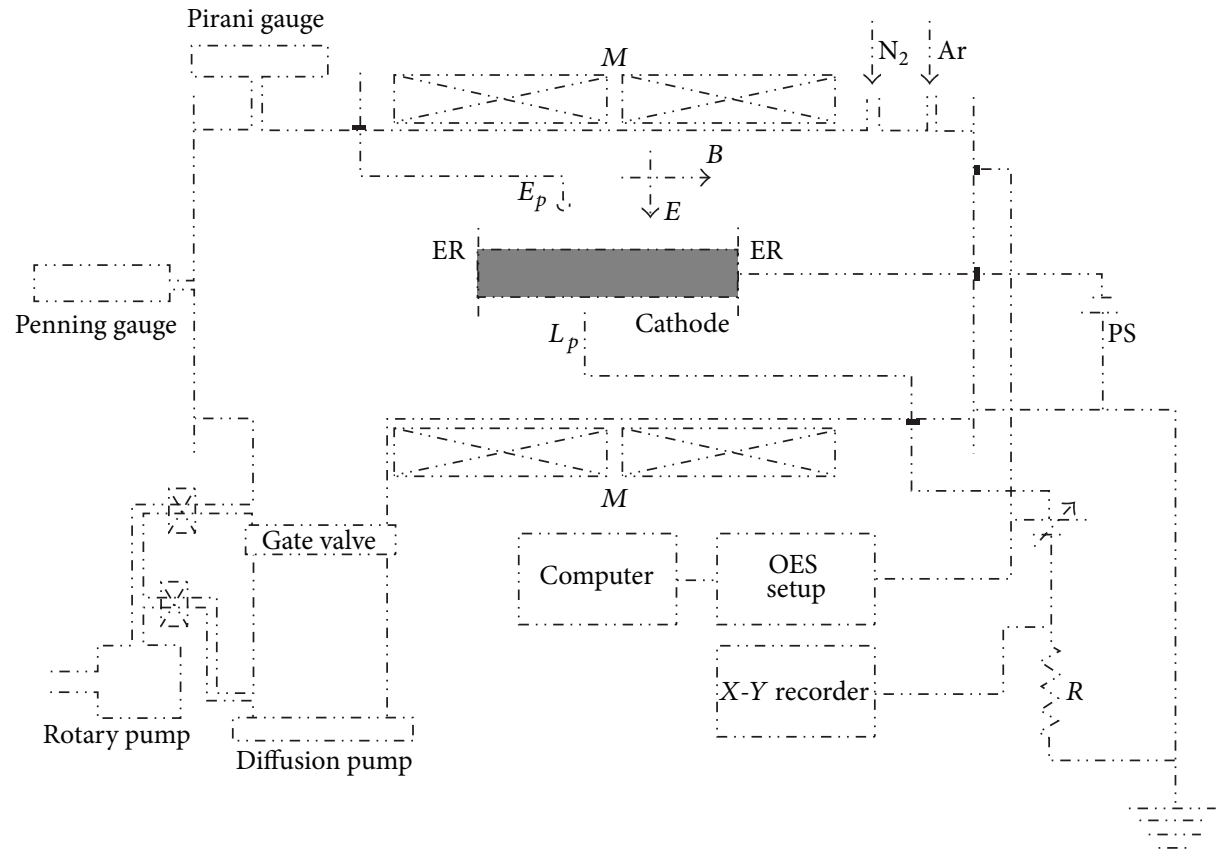

(a)

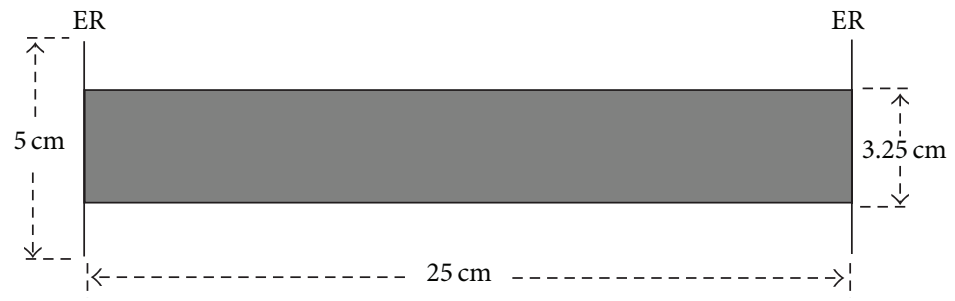

(b)

Figure 1: (a) Schematic diagram of the experimental set up: MM-magnetic field coils; B-magnetic field; E-electric field; Cathodetitanium/stainless steel target; PS—discharge power supply; ER-end reflectors; $L_{P}$-langmuir probe; $E_{P}$-emissive probe. (b) Schematic diagram of the titanium target cathode.

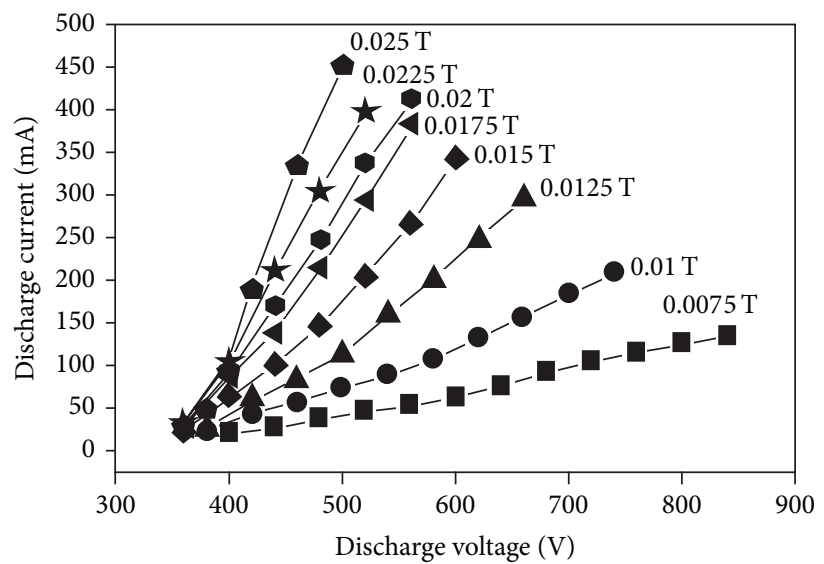

FIGURE 2: Discharge current-voltage characteristics at different magnetic fields. The total gas pressure is $2 \times 10^{-3}$ Torr at Ar: $\mathrm{N}_{2}=1: 1$. 


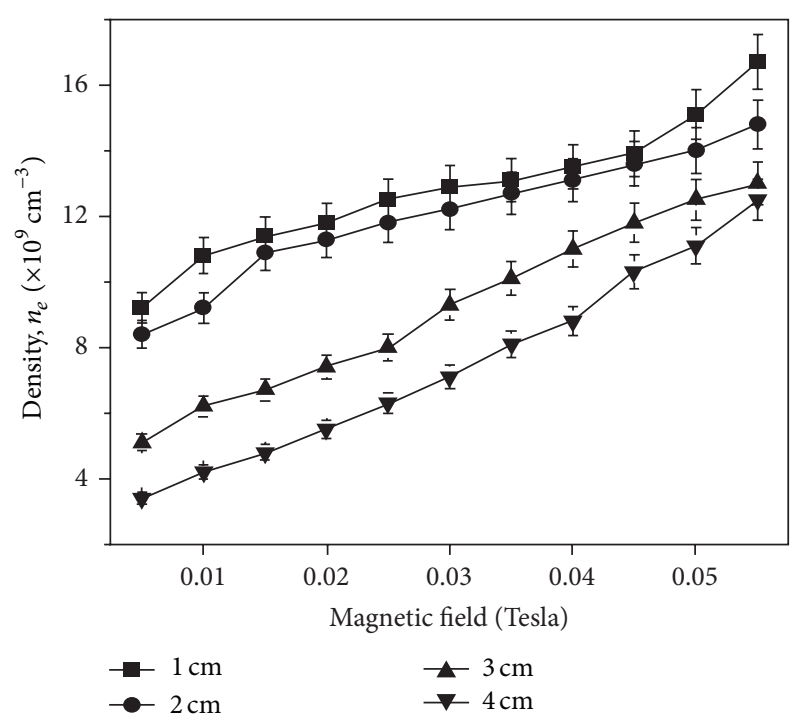

Figure 3: Dependence of plasma density on magnetic fields at different probe positions. The total gas pressure is $2 \times 10^{-3}$ Torr. Discharge voltage $=600 \mathrm{~V}$.

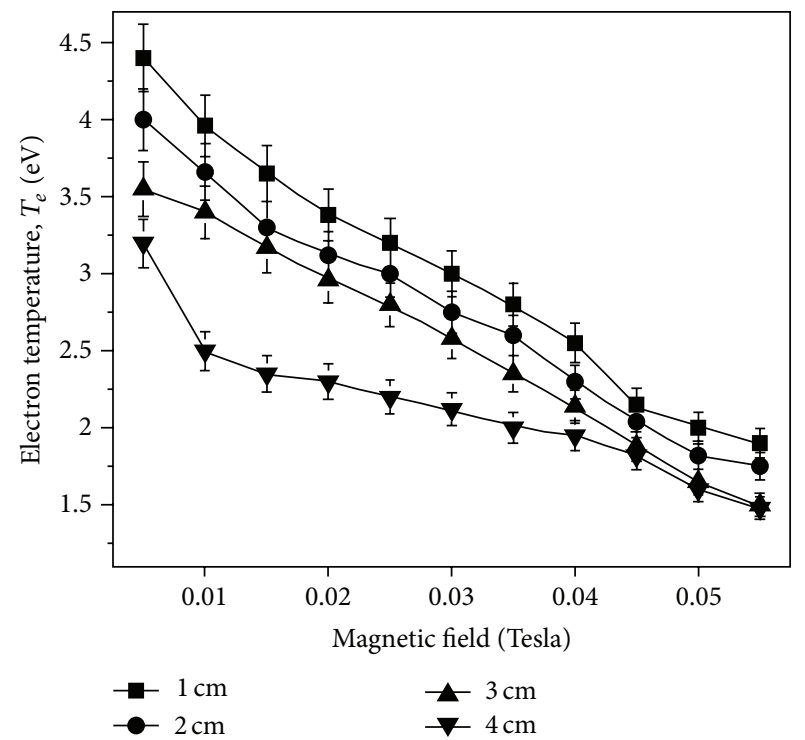

FIGURE 4: Electron temperature versus magnetic field at different probe positions. The total gas pressure is $2 \times 10^{-3}$ Torr. Discharge voltage $=600 \mathrm{~V}$.

In Figure 4, the variation of electron temperature measured at different magnetic fields for different positions of the Langmuir probe is shown. The discharge voltage is $600 \mathrm{~V}$ and the total gas pressure is $2 \times 10^{-3}$ Torr. The electron temperature is found to decrease from $\sim 4 \mathrm{eV}$ to $\sim 1.8 \mathrm{eV}$ with the increase of the magnetic field strength from 0.005 Tesla to 0.055 Tesla when it is measured at a distance of $2 \mathrm{~cm}$ from the cathode. When the magnetic field is increased, then it results in more number of electrons getting confined within the plasma, and these electrons lose their energy to a greater extent due to ionizing collisions during their drift due to $\mathbf{E} \times \mathbf{B}$

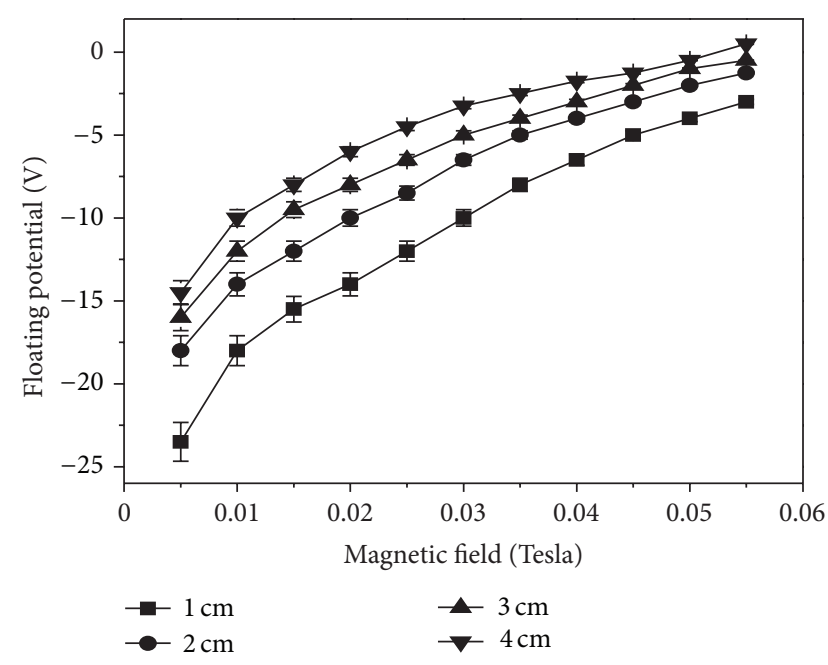

FIGURE 5: Floating potential versus magnetic field at different probe positions. The total gas pressure is $2 \times 10^{-3}$ Torr. Discharge voltage $=600 \mathrm{~V}$.

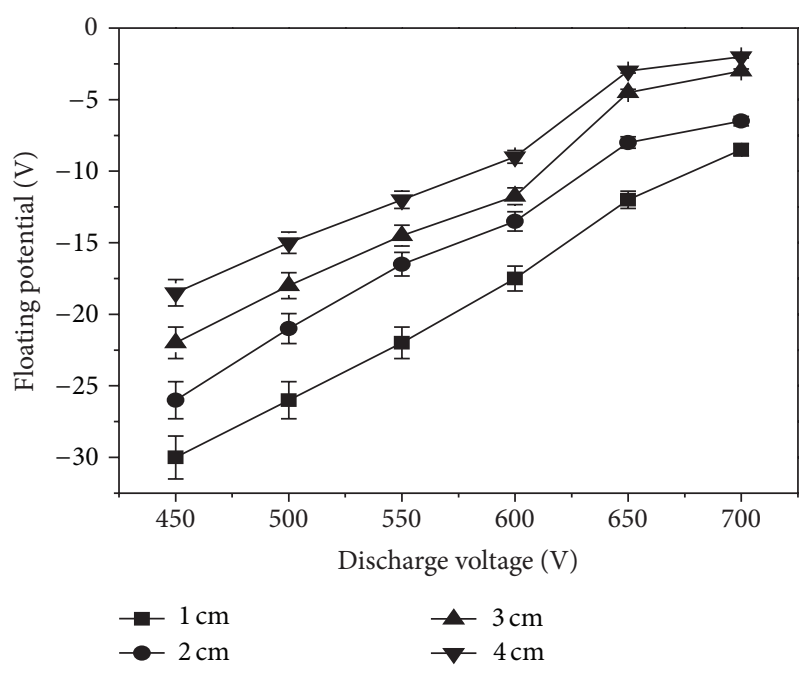

FIGURE 6: Floating potential versus discharge voltage at different probe positions. The total gas pressure is $2 \times 10^{-3}$ Torr. $\mathrm{B}=0.01$ Tesla.

effect. This leads to the decrease in the electron temperature value with the increase in the applied magnetic field strength. The electron temperature is higher near the cathode that is within the magnetic trap, and it goes on decreasing radially away from the cathode as the electrons lose their energy in collision and ionization while travelling radially outward. Near the cathode surface, the electron temperature is high and it decreases away from it. The high value of the electron temperature within the $\mathbf{E} \times \mathbf{B}$ trap is due to the confinement of the energetic electrons by the magnetic field. Electron temperature decreases towards the bulk plasma region when one moves away from the cathode surface.

The deposition of thin films is influenced by the floating potential. So, it becomes an important parameter during the thin film deposition process. In Figures 5 and 6, the corresponding variations of floating potential measured by 
TABLE 1

\begin{tabular}{|c|c|c|c|c|c|}
\hline Ar: $\mathrm{N}_{2}$ partial pressure ratio & $\Delta m(\mathrm{gm})$ & Area, $A\left(\mathrm{~cm}^{2}\right)$ & Density, $\rho\left(\mathrm{gcm}^{-3}\right)$ & Thickness, $t(\mu \mathrm{m})$ & $\begin{array}{l}\text { Thickness measured using } \\
\text { SEM }(\mu \mathrm{m})\end{array}$ \\
\hline $7: 1$ & $8.81 \times 10^{-4}$ & $72.25 \times 10^{-2}$ & 5.22 & 2.3 & \\
\hline $3: 1$ & $11.31 \times 10^{-4}$ & $72.25 \times 10^{-2}$ & 5.22 & 3 & 2.947 \\
\hline $5: 3$ & $14.55 \times 10^{-4}$ & $72.25 \times 10^{-2}$ & 5.22 & 3.9 & \\
\hline $1: 1$ & $18.86 \times 10^{-4}$ & $72.25 \times 10^{-2}$ & 5.22 & 5 & 4.860 \\
\hline $3: 5$ & $6.34 \times 10^{-4}$ & $72.25 \times 10^{-2}$ & 5.22 & 1.7 & \\
\hline $1: 3$ & $1.89 \times 10^{-4}$ & $72.25 \times 10^{-2}$ & 5.22 & 0.5 & 0.507 \\
\hline $1: 7$ & $1.16 \times 10^{-4}$ & $72.25 \times 10^{-2}$ & 5.22 & 0.3 & \\
\hline
\end{tabular}

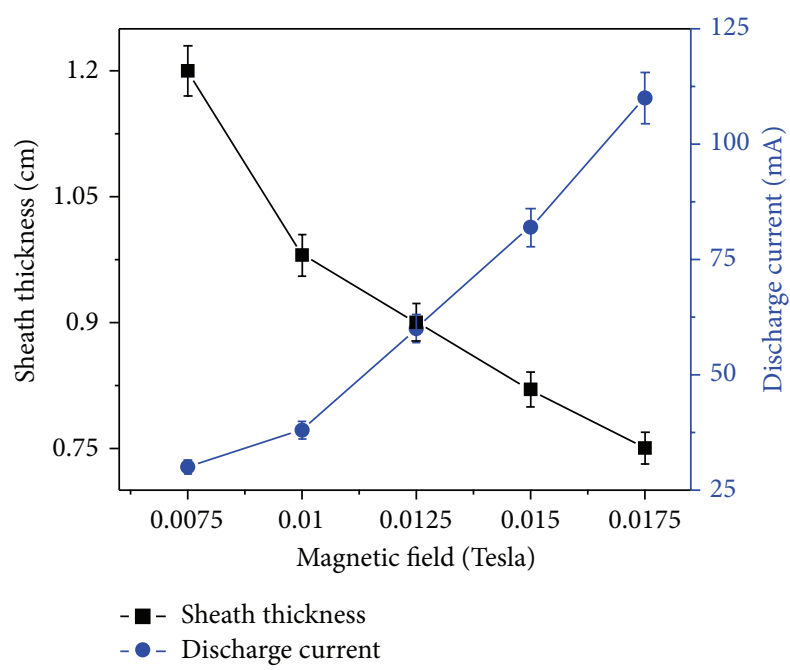

FIGURE 7: Sheath thickness and discharge current variation with magnetic field at discharge voltage $=600 \mathrm{~V}$ and $\mathrm{P}_{\text {Ar- } \mathrm{N}_{2}}=2 \times$ $10^{-3}$ Torr.

the Langmuir probe at different distances away from the cathode for different discharge voltages and magnetic fields, respectively, are shown. Floating potential takes less negative values with the increase of both the discharge voltage and the magnetic field. At constant discharge voltage, floating potential increases (less negative) with increasing radial distance.

Figure 7 represents the variation of sheath thickness and discharge current with magnetic field as a parameter at fixed argon-nitrogen total pressure of $2 \times 10^{-3}$ Torr and discharge voltage of $600 \mathrm{~V}$. With increasing magnetic field, the plasma potential is found to decrease. At higher magnetic fields, the electrons become more and more confined, and therefore the plasma potential becomes more negative to control the ion loss rate and maintain the quasineutrality of the plasma. For all the magnetic fields, a sharp radial gradient in the plasma potential and thus strong electric field is present near the cathode region resulting in the formation of the sheath. The enhanced confinement of electrons near the cathode surface will reduce the effective positive ion concentration within the sheath. This should effectively result in the expansion of the cathode sheath. On the contrary, there occurs an interesting observation in the sheath thickness when magnetic field is increased. The cathode sheath is found to contract with the increase in the magnetic field. The increase in the overall plasma density is responsible for the decrease in the cathode sheath thickness. Here, the plasma density influence on the nature of the cathode sheath thickness is more dominating than the electron confinement factor.

For quality deposition of thin films, it is necessary to optimize the magnetron sputtering by maintaining the argon and reactive gas (nitrogen) partial pressures. This variation in the argon and nitrogen partial pressures influences the plasma sheath, which is significant in the ionization process as well as the plasma transport mechanism of the discharge. It has been observed that the space charge density in the cathode sheath and the current density to the cathode decrease when nitrogen concentration is increased. With the increase in sheath thickness, the strength of the sheath electric field decreases which in turn will lead to the lowering of the energy of the ions bombarding the cathode resulting in low rate of sputtering.

The corresponding deposition rate for different $\mathrm{Ar}: \mathrm{N}_{2}$ partial pressure ratios (Ar: $\mathrm{N}_{2} \sim 7: 1, \operatorname{Ar}: \mathrm{N}_{2} \sim 3: 1, \operatorname{Ar}: \mathrm{N}_{2} \sim$ $5: 3, \operatorname{Ar}: \mathrm{N}_{2} \sim 1: 1, \operatorname{Ar}: \mathrm{N}_{2} \sim 3: 5, \operatorname{Ar}: \mathrm{N}_{2} \sim 1: 3$, and $\operatorname{Ar}: \mathrm{N}_{2} \sim$ $1: 7)$ have been measured. The deposition condition of the TiN films is as follows-discharge voltage is $600 \mathrm{~V}$, magnetic field is 0.01 Tesla, and total gas pressure is $2 \times 10^{-3}$ Torr. The deposition is done for time duration of 75 minutes under controlled discharge parameters. The thickness $(t)$ of the deposited film is calculated from the relation given as follows:

$$
t=\frac{\Delta m}{\rho \times A},
$$

where $\Delta m, \rho$, and $A$ are the deposited mass, bulk density of TiN and surface area of the substrate, respectively. The corresponding thickness values of the deposited TiN thin films at different $\mathrm{Ar}: \mathrm{N}_{2}$ partial pressure ratios are shown in Table 1. Keeping in mind the fact that the deposited film thickness values shown in the table have been obtained by considering the theoretical bulk density of TiN, we have also determined the thickness of the film using Scanning Electron Microscopy (SEM) technique. The thickness of the films measured using SEM at three different $\mathrm{Ar}: \mathrm{N}_{2}$ partial pressure ratios of $3: 1,1: 1$, and $1: 3$ are $2.947 \mu \mathrm{m}, 4.860 \mu \mathrm{m}$, and $0.507 \mu \mathrm{m}$, respectively. These values are comparable to the thickness obtained using the measured deposited mass 


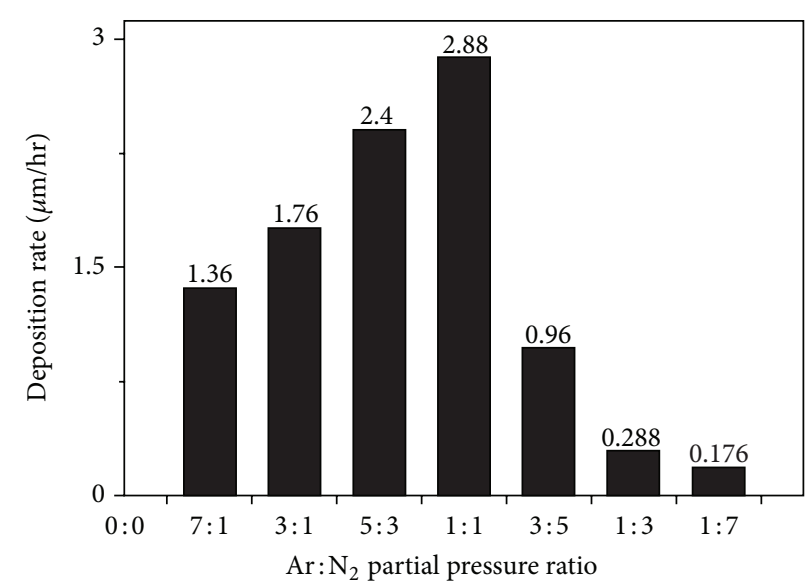

FIGURE 8: Bar chart showing TiN film deposition rate for different Ar: $\mathrm{N}_{2}$ partial pressure ratios $\left(\mathrm{Ar}: \mathrm{N}_{2} \sim 7: 1, \operatorname{Ar}: \mathrm{N}_{2} \sim 3: 1, \operatorname{Ar}: \mathrm{N}_{2} \sim\right.$ 5:3, Ar: $\mathrm{N}_{2} \sim 1: 1, \operatorname{Ar}: \mathrm{N}_{2} \sim 3: 5, \operatorname{Ar}: \mathrm{N}_{2} \sim 1: 3$, and $\left.\operatorname{Ar}: \mathrm{N}_{2} \sim 1: 7\right)$ at fixed total pressure $2 \times 10^{-3}$ Torr. Discharge voltage is $600 \mathrm{~V}$ and magnetic field is 0.01 Tesla.

using the previous equation. Figure 8 shows the deposition rates for the different Ar: $\mathrm{N}_{2}$ partial pressure ratios $\left(\right.$ Ar $: \mathrm{N}_{2} \sim$ 7:1, Ar: $\mathrm{N}_{2} \sim 3: 1$, Ar: $\mathrm{N}_{2} \sim 5: 3, \operatorname{Ar}: \mathrm{N}_{2} \sim 1: 1$, Ar: $\mathrm{N}_{2} \sim$ 3:5, Ar: $\mathrm{N}_{2} \sim 1: 3$, and Ar: $\mathrm{N}_{2} \sim 1: 7$ ) in the gas mixture. The deposition rate has been calculated from the amount of deposited mass of the film per hour over the substrate. The rate of formation of $\mathrm{TiN}$ depends on the amount of titanium atoms being sputtered from the target and the rate at which they get nitrided by reacting with nitrogen. There is a gradual increase in the reaction rate between titanium and nitrogen atoms with increase of nitrogen gas and the simultaneous decrease of argon gas in the gas mixture, which results in the increase of the rate of deposition. It has been observed that Ar: $\mathrm{N}_{2} \sim 1: 1$ in the gas mixture is the most suitable condition for high-rate TiN film deposition. At this particular condition, the sputtering rate balances the reaction rate between titanium and nitrogen atoms. As a result, almost all the sputtered titanium atoms from the target react with the nitrogen gas atoms to form $\mathrm{TiN}$, and the deposition rate becomes maximum at this condition. As argon is heavier than nitrogen, so it is mainly responsible for the sputtering. Decrease in argon ion density reduces the sputtering rate of titanium atoms.

\section{Conclusion}

This paper contains the experimental results of the study on the discharge characteristics, for example, current-voltage characteristics, plasma density, electron temperature, and floating potential in a direct current cylindrical magnetron discharge plasma in relation to material processing applications, namely, thin film deposition over substrate. Application of the magnetic field in such system increases the electron confinement and results in the increase of the rate of ionization. This enhances the rate of sputtering of the metal target. Thin film coating of titanium nitride has been deposited on bell-metal in reactive discharge environment using nitrogen, and the film coating has been very effective in providing good surface protection.

\section{Acknowledgments}

This work was supported by a grant from the Department of Science and Technology, Government of India. The author thanks the entire research group of the Material Sciences Division, Institute of Advanced Study in Science and Technology (IASST), Guwahati, for their help and support during the author's working tenure at that division.

\section{References}

[1] B. Subramanian, C. V. Muraleedharan, R. Ananthakumar, and M. Jayachandran, "A comparative study of titanium nitride (TiN), titanium oxy nitride (TiON) and titanium aluminum nitride (TiAlN), as surface coatings for bio implants," Surface and Coatings Technology, vol. 205, no. 21-22, pp. 5014-5020, 2011.

[2] A. Grill, Cold Plasma in Materials Fabrication, IEEE, New York, NY, USA, 1994.

[3] N. Kumari, P. S. Das, N. K. Joshi, and P. K. Barhai, "Correlations of plasma parameters and properties of magnetron sputtered TiN films," The European Physical Journal, vol. 59, no. 2, Article ID 20302, 7 pages, 2012.

[4] J. A. Thornton, "Magnetron sputtering: basic physics and application to cylindrical magnetrons," Journal of Vacuum Science and Technology, vol. 15, no. 2, pp. 171-177, 1978.

[5] S. Z. Wu, "Dependence of plasma characteristics on dc magnetron sputter parameters," Journal of Applied Physics, vol. 98, Article ID 083301, 5 pages, 2005.

[6] T. E. Sheridan, M. J. Goeckner, and J. Goree, "Model of energetic electron transport in magnetron discharges J," Journal of Vacuum Science \& Technology A, vol. 8, no. 30, 8 pages, 1990.

[7] S. L. Rohde, I. Petrov, W. D. Sproul, S. A. Barnett, P. J. Rudnik, and M. E. Graham, "Effects of an unbalanced magnetron in a unique dual-cathode, high rate reactive sputtering system," Thin Solid Films, vol. 193-194, no. 1, pp. 117-126, 1990.

[8] R. P. Howson, H. A. J'Afer, and A. G. Spencer, "Substrate effects from an unbalanced magnetron," Thin Solid Films, vol. 193-194, no. 1, pp. 127-137, 1990.

[9] S. M. Borah, H. Bailung, A. R. Pal, and J. Chutia, "Study on the influence of nitrogen on titanium nitride in a dc post magnetron sputtering plasma system," Journal of Physics D, vol. 41, no. 19, Article ID 195205, 2008.

[10] S. M. Borah, A. R. Pal, H. Bailung, and J. Chutia, "Optimization of plasma parameters for high rate deposition of titanium nitride films as protective coating on bell-metal by reactive sputtering in cylindrical magnetron device," Applied Surface Science, vol. 254, no. 18, pp. 5760-5765, 2008. 

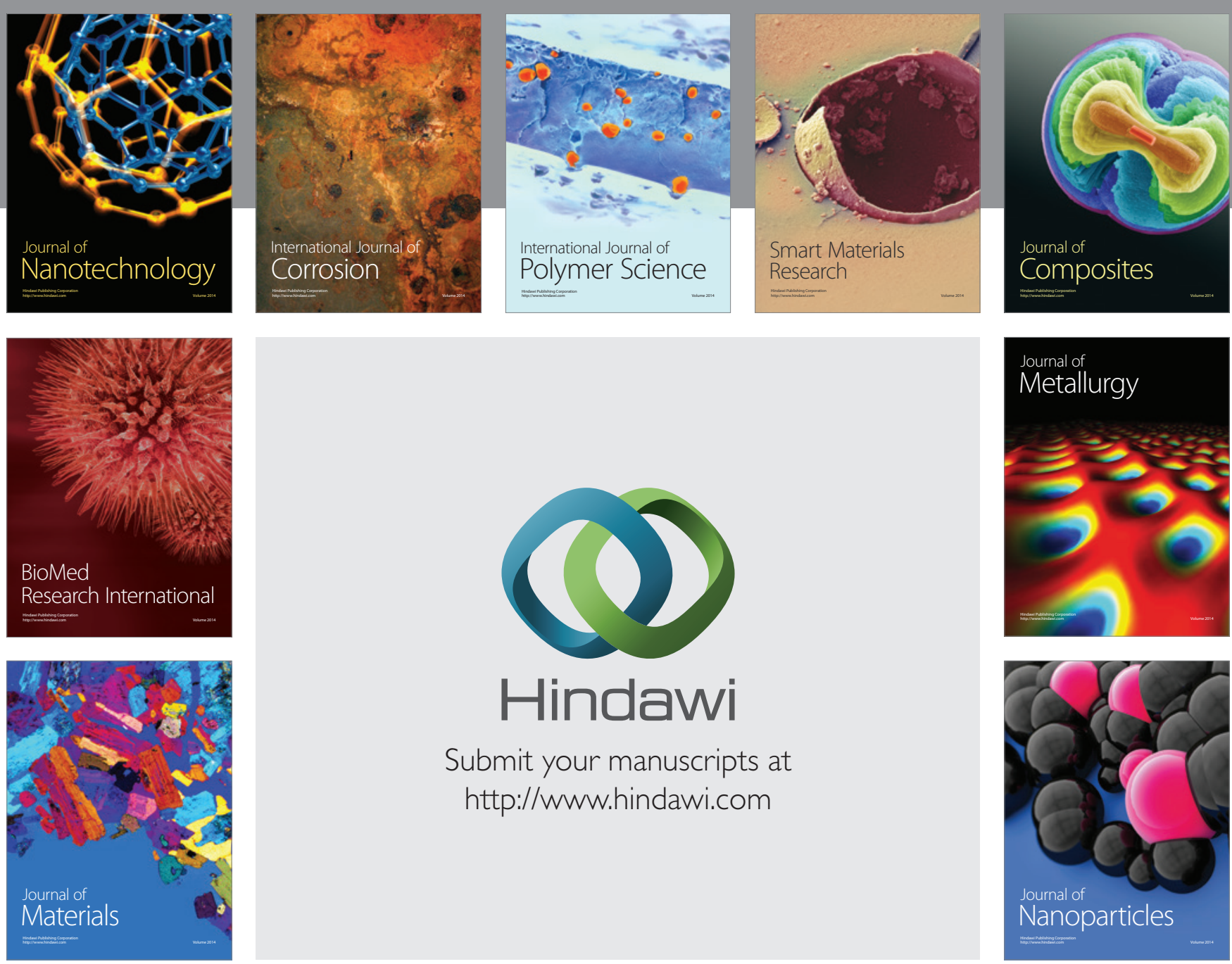

Submit your manuscripts at http://www.hindawi.com
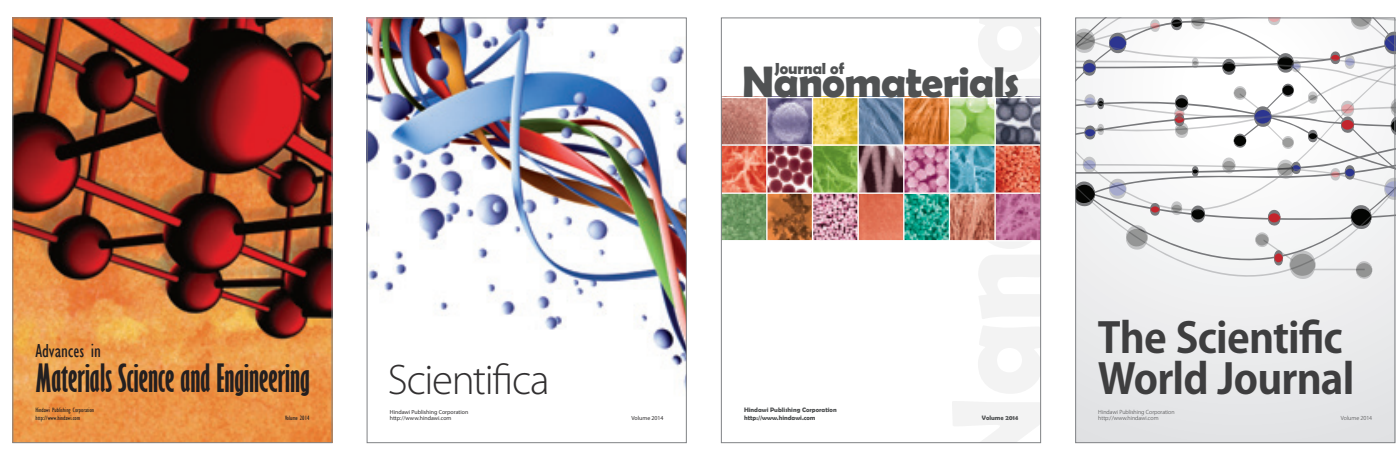

\section{The Scientific World Journal}
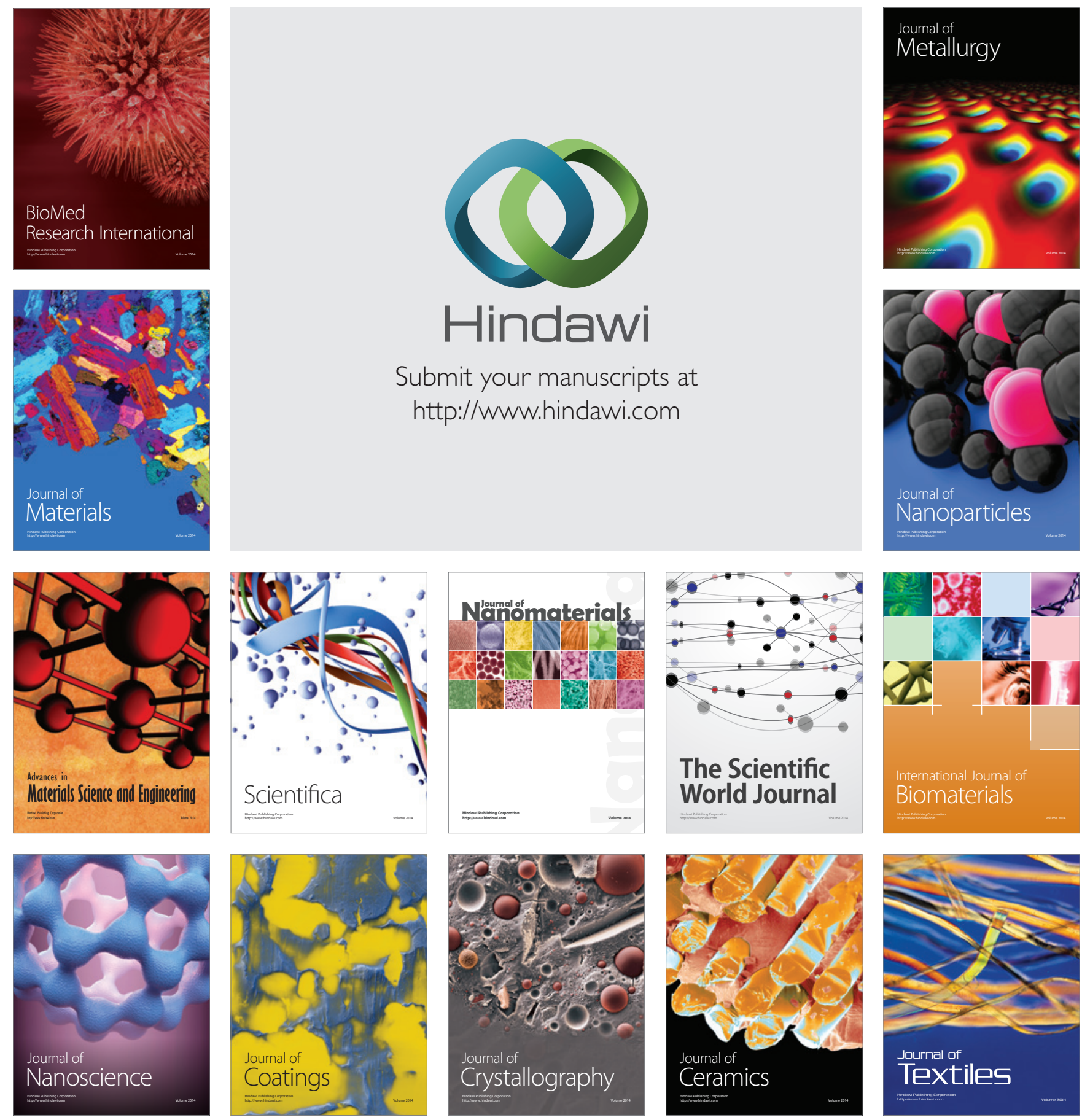